\title{
Desarrollo larvario y supervivencia hasta la metamorfosis del erizo Tripneutes ventricosus (Lamarck) (Echinodermata: Echinoidea) alimentado con microalgas a dos temperaturas
}

\author{
Larval development and survival to metamorphosis of sea urchin Tripneutes ventricosus (Lamarck) \\ (Echinodermata: Echinoidea) fed on microalgae at two temperatures \\ Jesús Rosas ${ }^{1}$, Aidé Velásquez $^{2 *}$, Saccha Fernández ${ }^{2}$, Ernesto Mata ${ }^{1}$ y Tomas Cabrera ${ }^{2}$ \\ ${ }^{1}$ Instituto de Investigaciones Científicas, Universidad de Oriente, Núcleo de Nueva Esparta, Boca de Río, \\ Estado Nueva Esparta, Venezuela, Apartado Postal 788, Porlamar, Isla de Margarita \\ ${ }^{2}$ Escuela de Ciencias Aplicadas del Mar, Universidad de Oriente, Núcleo de Nueva Esparta, Boca de Río, \\ Estado Nueva Esparta, Venezuela, Apartado Postal 788, Porlamar, Isla de Margarita \\ *aijuaa@gmail.com
}

\begin{abstract}
Larval development and survival of Tripneutes ventricosus up to metamorphosis were evaluated. Ten sexually mature specimens were collected from 'Faro de Charagato' to 'Laja Amarilla' (1050’07"N, 6409'33"O) Cubagua island, Nueva Esparta state, Venezuela. Spontaneous spawning of the organisms was produced during their transport to the laboratory. Pluteus larvae ( 2 ind $\left.\mathrm{mL}^{-1}\right)$ were randomly distributed into 16 plastic recipients (18 L) (32000 larvae/recipient), with $16 \mathrm{~L}$ of filtered sea water and continuous aeration. Two groups of eight recipients each were set up; one group was kept at outdoor temperature $\left(29 \pm 1{ }^{\circ} \mathrm{C}\right)$ and the other one at laboratory temperature $\left(23 \pm 2^{\circ} \mathrm{C}\right)$. For each temperature Chaetoceros gracilis ( 50000 cells $\mathrm{mL}^{-1}$ ) was added as food to four recipients and a mixture of C. gracilis plus Isochrysis galbana (25000 cells $\mathrm{mL}^{-1}$ of each species) to the other four recipients. Survival was daily determined, from 4-mL sample taken from each treatment, and the live larvae were quantified. The body length was estimated every other day in five preserved larvae with $5 \%$ formaldehyde. Postlarval stage was attained 24 days after spawning in both culture temperatures. Morphological differences were not detected neither body length between treatments, however, survival showed differences (ANOVA, $P<0.05)$ with the best results in larvae fed on $C$. gracilis and $I$. galbana mixture $(30 \pm 0.30 \%)$ at outdoor temperature.
\end{abstract}

Key words: Morphometric, diets, experimental culture
Resumen.- Se evaluó el desarrollo larvario y la supervivencia del erizo Tripneutes ventricosus hasta la metamorfosis. Diez ejemplares sexualmente maduros fueron recolectados entre el Faro de Charagato hasta la Laja Amarilla (1050'07"N, 6409'33"O) en la Isla de Cubagua, Estado Nueva Esparta, Venezuela. Se produjo el desove espontáneamente durante el traslado hasta el laboratorio. Larvas pluteus $\left(2\right.$ ind $\left.\mathrm{mL}^{-1}\right)$ se distribuyeron aleatoriamente en 16 envases de plástico de $18 \mathrm{~L}$ (32000 larvas/recipiente), con $16 \mathrm{~L}$ de agua de mar filtrada y aireación continua. Se trabajó con dos grupos de ocho envases, uno mantenido a temperatura exterior $\left(29 \pm 1^{\circ} \mathrm{C}\right)$ y el otro a temperatura del laboratorio $\left(23 \pm 2^{\circ} \mathrm{C}\right)$. Para cada temperatura, se utilizó Chaetoceros gracilis $\left(50000\right.$ cél $\left.\mathrm{mL}^{-1}\right)$ en cuatro recipientes y la mezcla de C. gracilis más Isochrysis galbana ( 25000 cél $\mathrm{mL}^{-1}$ de cada especie) en los otros cuatro recipientes. La supervivencia se determinó diariamente, tomando cuatro alícuotas de $1 \mathrm{~mL}$ por cada tratamiento y cuantificando las larvas vivas. La longitud del cuerpo se determinó cada tercer día en cinco larvas fijadas con formalina al $5 \%$. La fase postlarval se alcanzó a los 24 días después del desove tanto a temperatura del laboratorio como del exterior. No se detectaron diferencias morfológicas ni en la longitud del cuerpo entre los tratamientos, sin embargo, la supervivencia mostró diferencias (ANOVA, $P<0,05)$ con los mejores valores en larvas alimentadas con la mezcla de C. gracilis e I. galbana $(30 \pm 0,30 \%)$ a temperatura exterior.

Palabras clave: Morfometría, dietas, cultivo experimental

\section{Introducción}

Los estudios ecológicos y comerciales referidos a erizos de diferentes especies están más desarrollados en países como Chile, donde se cultivan masivamente juveniles del erizo Loxechinus albus (Molina) en el laboratorio. Bustos $\&$ Olave (2001) realizaron un manual para el cultivo del erizo L. albus, y Lawrence et al. (2001) estudiaron la ecología de Tripneutes ventricosus (Lamarck). Pacheco (1998) desarrolló en Chile investigaciones científicotécnicas de cultivo del erizo L. albus en sistemas semicerrados y Eckert (1998) estudió el desarrollo, crecimiento y morfología en el erizo de mar Diadema antillarum Philippi en Florida. 
En Venezuela, existen tres especies de erizo de mar con potencial de cultivo: Lytechinus variegatus (Lamarck), Echinometra lucunter (Linnaeus) y Tripneutes ventricosus, ya que reúnen tres características importantes, el rápido crecimiento, la capacidad para producir grandes cantidades de gónadas en corto tiempo y su adaptación al cautiverio. La crisis económica en Venezuela plantea a corto plazo la explotación artesanal o industrial de estos recursos, como ha ocurrido en Chile, Estados Unidos, Japón, Francia y Australia. Lo anterior pondría en riesgo el recurso debido a un posible colapso de los bancos naturales, por lo que realizar estudios relacionados con la producción y el cultivo de larvas de estas especies en condiciones controladas resulta de suma importancia para atenuar la presión sobre el recurso (Gómez \& Gómez 2005).

El erizo Tripneutes ventricosus conjuntamente con las demás especies presentes en aguas circundantes de la Isla de Margarita, representa a mediano plazo una fuente importante para la explotación (Gómez \& Gómez 2005), lo cual hace necesario que se estudien aspectos relacionados con su ecología, índice gonadosomático y distribución a fin de evitar una sobre explotación del recurso. Lawrence \& Lawrence (2003) refieren la importancia económica de los erizos y sugirieron realizar ensayos concernientes a la cría de larvas en condiciones de laboratorio, que pudieran ser destinadas a repoblar aquellas zonas diezmadas por la excesiva explotación y para incentivar los cultivos en regiones con estudios ambientales preestablecidos.

Tripneutes ventricosus habita una gran diversidad de fondos que van desde áreas de pastos, áreas profundas, hasta arrecifes de coral entre rocas y escombros (Gordon et al. 1995). Se alimentan de fanerógamas marinas como Thalassia hemprichii (Ehrenb. Ex Solms) Asch., Syringodium isoelifolium (Aschers.), microalgas y macroalgas tales como Halimeda entre otras y corales cuando están disponibles (Klumpp et al. 1993).

En las islas de Margarita y Coche, en Venezuela, se han realizado investigaciones sobre los erizos Lytechinus variegatus, Echinometra lucunter y Arbacia punctulata (Lamarck) aunque en la zona existen por lo menos ocho especies (Gómez 2000).

Debido a que $T$. ventricosus es una especie potencial para el cultivo y con la que no se han realizado trabajos de investigación, en el presente estudio se planteó establecer el tiempo de desarrollo larval y supervivencia hasta la metamorfosis a temperatura exterior $\left(29 \pm 1^{\circ} \mathrm{C}\right)$ y de laboratorio $\left(23 \pm 2^{\circ} \mathrm{C}\right)$, alimentados con Chaetoceros gracilis y la mezcla de C. gracilis e Isochrysis galbana.

\section{Material y métodos}

Se recolectaron manualmente 10 organismos sexualmente maduros (6 a 7,5 cm de alto y de 11 a $15 \mathrm{~cm}$ de diámetro de testa) de $T$. ventricosus en la zona comprendida desde el Faro de Charagato hasta la Laja Amarilla (1050'07"N, $64^{\circ} 09^{\prime} 33^{\prime \prime W}$ ) en la Isla de Cubagua, Estado Nueva Esparta. Las condiciones en el lugar de muestreo fueron de $26^{\circ} \mathrm{C}$ y $36 \mathrm{psu}$. Los erizos fueron lavados individualmente con agua de mar filtrada antes de ser trasladados al Laboratorio de Cultivo en el Instituto de Investigaciones Científicas (IIC) de la Universidad de Oriente, Núcleo de Nueva Esparta, Venezuela.

Los organismos fueron transportados en envases separados, con agua de mar filtrada; durante el traslado hasta el laboratorio de cultivo se produjo el desove espontáneamente, quedando los gametos inmersos en el agua de mar filtrada, de la cual se tomaron muestras para observar la presencia de gametos masculinos o femeninos. Los gametos femeninos fueron filtrados a través de un tamiz de $120 \mu \mathrm{m}$, y colocados en un recipiente de vidrio de $5 \mathrm{~L}$, con $3 \mathrm{~L}$ de agua de mar filtrada y aireación moderada. En el caso de gametos masculinos, se seleccionaron los espermatozoides más activos (Eckert 1998) y se agregaron al recipiente con óvulos para fertilizar (100:1 espermatozoides/óvulo) de acuerdo al criterio de Grosjean et al. (1998).

Después de la fecundación, grupos de 100.000 huevos fueron colocados en recipientes de $18 \mathrm{~L}$ con $16 \mathrm{~L}$ de agua de mar filtrada por $24 \mathrm{~h}$ a temperaturas de 23 y $29^{\circ} \mathrm{C}$. A las 32 horas después de la fertilización se obtuvieron larvas pluteus, las cuales se distribuyeron aleatoriamente (2 larvas $\mathrm{mL}^{-1}$ ) en 16 envases de plástico de $18 \mathrm{~L}$ (32000 larvas/recipiente), con $16 \mathrm{~L}$ de agua de mar filtrada $\mathrm{y}$ aireación continua. El grupo de envases fue dividido en dos, ocho fueron mantenidos a la temperatura del exterior $\left(29 \pm 1^{\circ} \mathrm{C}\right)$ y el otro a temperatura del laboratorio $(23 \pm$ $2^{\circ} \mathrm{C}$ ). A las larvas contenidas en cuatro recipientes se les suministró Chaetoceros gracilis (50000 cél mL-1) y a los otros cuatro envases se le suministró la mezcla de $C$. gracilis e Isochrysis galbana (25000 cél $\mathrm{mL}^{-1}$ de cada especie). El procedimiento se realizó para ambas temperaturas. Las larvas desde pluteus con dos brazos hasta que tuvieron cuatro brazos fueron alimentadas con las microalgas antes mencionadas en base a los trabajos realizados por otros autores, quienes emplearon las mismas especies y concentraciones en el cultivo larval de Echinometra lucunter (Astudillo et al. 2005).

Las temperaturas se seleccionaron en base al promedio de la variación térmica del sitio de recolección de los erizos adultos, lo cual derivó en la selección de un 


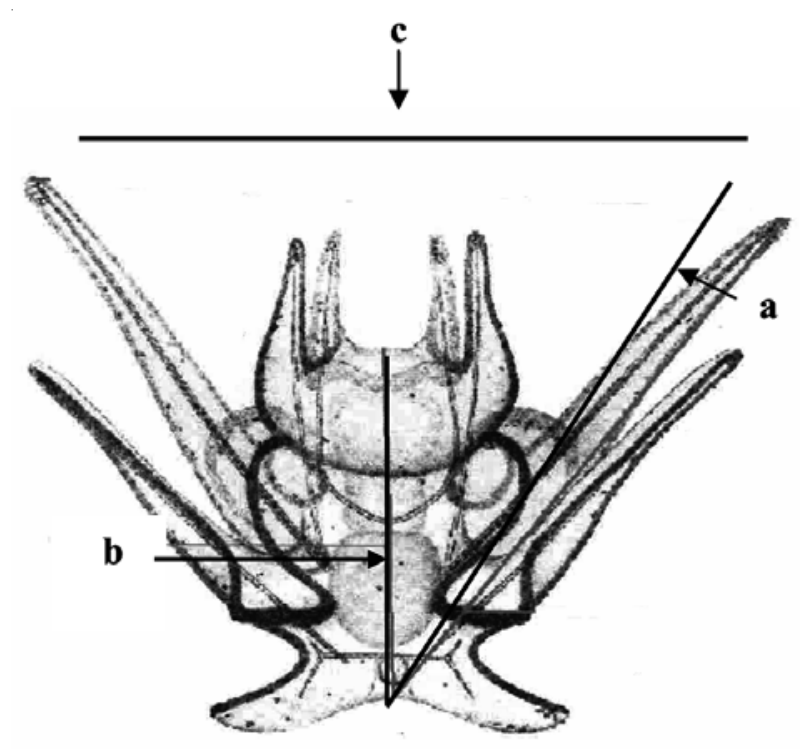

Figura 1

\begin{abstract}
Medidas ( $\mu \mathrm{m})$ tomadas: (a) longitud del cuerpo considerando los brazos, (b) longitud media del cuerpo sin considerar los brazos y (c) distancia entre las aberturas de ambos brazos
\end{abstract}

Measurements ( $\mu \mathrm{m})$ taken: (a) Body length considering arms, (b) median body length without arms and (c) distance between arms opening

intervalo de 20 a $30^{\circ} \mathrm{C}$ aproximadamente y también para tratar de disminuir la carga bacteriana presente en los cultivos de larvas, la cual fue menor a temperatura del laboratorio y a 30 ups.

La cantidad y tipo de alimento (C. gracilis a 50.000 cél $\mathrm{mL}^{-1}$ y la mezcla de Chaetoceros gracilis a 25000 cél $\mathrm{mL}^{-1}$

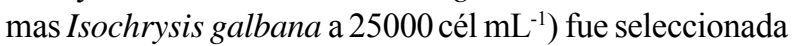
según los criterios de Young \& George (2000), incrementando la concentración entre un 10 y $15 \%$ a medida que la larvas crecieron (Bustos \& Olave 2001). Diariamente, en todos los recipientes de cultivo se cambió el $75 \%$ del volumen total de agua, se removió la materia orgánica depositada en el fondo y se añadió el alimento. Semanalmente las larvas fueron cambiadas a un nuevo recipiente hasta que alcanzaron el estadio de competencia (Domínguez et al. 2007).

Para estimar la supervivencia, diariamente se tomaron cuatro alícuotas de $1 \mathrm{~mL}$ de cada réplica de cultivo, cuantificándose las larvas vivas con ayuda de una cámara Bogorov y lupa estereoscópica a 50X de aumento. Los datos obtenidos para cada estadio se estimaron en base a un $100 \%$ de supervivencia. Las medidas de la longitud del cuerpo $(\mu \mathrm{m})$ se tomaron desde el ápice al extremo opuesto donde comienza el cuerpo (Fig. 1a), la longitud media del cuerpo sin considerar los brazos (Fig. 1b) y la distancia entre las aberturas de los dos primeros brazos (Fig. 1c) de cinco larvas fijadas con formalina al 5\% (Eckert 1998). Las diferencias entre los valores supervivencia y longitud del cuerpo en los diferentes tratamientos se establecieron con un análisis de varianza (ANOVA) de factores múltiples. En los tratamientos donde se registraron diferencias se aplicaron pruebas $a$ posteriori LSD (Sokal \& Röhlf 1981).

\section{Resultados}

\section{Período precompetente}

El desarrollo larval del erizo Tripneutes ventricosus se inició ocho horas después de la fertilización, con la fase prisma. Transcurridas 32 horas desde la fertilización adoptó el estadio de pluteus con 2 brazos, el lado ventral de la larva fue convirtiéndose en una superficie oral aplanada y la zona dorsal se convirtió en una región abultada y curva. Un par de varas a cada lado del intestino conectado por una vara transversal constituyeron la primera evidencia visible del esqueleto; el movimiento de las larvas fue giratorio y la coloración del estómago se relacionó con el color del alimento ingerido.

Los mayores valores promedios de longitud del cuerpo $\mathrm{y}$ abertura de los brazos de las larvas fueron $185 \pm 6,05 \mathrm{y}$ $220 \pm 16,24 \mu \mathrm{m}$ con C. gracilis y $172 \pm 13,05$ y $200 \pm$ $12,08 \mu \mathrm{m}$ con la mezcla de C. gracilis e I. galbana a temperatura del laboratorio, mientras que a temperatura del exterior la longitud del cuerpo y abertura de los brazos de las larvas alimentadas con C. gracilis fue de $195 \pm$ 7,15 y $225 \pm 18,11 \mu \mathrm{m}$ y con la mezcla de $182 \pm 12,15$ y $210 \pm 11,05 \mu \mathrm{m}$ respectivamente (Tabla 1 ).

El mayor porcentaje de supervivencia de los erizos fue de $61 \%$ aproximadamente, al tercer día, en ambas dietas y en las dos temperaturas, detectándose una disminución del 39\% respecto al inicio del cultivo.

El sexto día después de la fertilización, en los tratamientos a ambas temperaturas, se registraron larvas de cuatro brazos, que sobresalían desde el lóbulo oral (Fig. 2A). Se inició el desarrollo de un par de protuberancias en ambos lados en la parte inferior y se inició la formación del tercer par de brazos.

La longitud del cuerpo de las larvas en los tratamientos a temperatura del laboratorio y alimentadas con C. gracilis fue de $314,30 \pm 23,30 \mu \mathrm{m}$ y la abertura de los brazos de $315,05 \pm 26,10 \mu \mathrm{m}$, mientras que en las larvas alimentadas con la mezcla de microalgas fue de $370 \pm 20,00$ y $356 \pm 15,10 \mu \mathrm{m}$ respectivamente. En el caso de las larvas 


\section{Tabla 1}

Longitud del cuerpo $(\mu \mathrm{m})$, abertura de los brazos $(\mu \mathrm{m})$ y supervivencia $(\%)$ de los diferentes estadios de Tripneutes ventricosus alimentados con $C$. gracilis y la mezcla de C. gracilis + I. galbana a temperaturas del laboratorio y exterior

Body length $(\mu \mathrm{m})$, arms aperture $(\mu \mathrm{m})$ and survival (\%) of different stages of Tripneutes ventricosus larvae fed on C. gracilis and a mixture of C. gracilis and I. galbana to laboratory and outdoor temperature

\begin{tabular}{|c|c|c|c|c|c|}
\hline Estadio & Supervivencia & Longitud del cuerpo & Abertura de brazos & Dieta & Temperatura \\
\hline $\begin{array}{l}\text { Prisma } \\
\text { (8 horas) }\end{array}$ & 100,00 & & & Ambas & Ambas \\
\hline \multirow{4}{*}{$\begin{array}{l}\text { Pluteus dos brazos } \\
\text { ( } 32 \text { horas) }\end{array}$} & & $185,00 \pm 6,05$ & $220,00 \pm 16,24$ & C. gracilis & Laboratorio \\
\hline & & $172,00 \pm 13,05$ & $200,00 \pm 12,08$ & Mezcla & Laboratorio \\
\hline & & $182,00 \pm 12,15$ & $210,00 \pm 11,05$ & C. gracilis & Exterior \\
\hline & & $195,00 \pm 7,15$ & $225,00 \pm 18,11$ & Mezcla & Exterior \\
\hline (Tres días) & $61 \pm 0,00$ & & & Ambas & Ambas \\
\hline \multirow{4}{*}{$\begin{array}{l}\text { Pluteus } 4 \text { brazos } \\
\quad \text { ( } 6 \text { días) }\end{array}$} & & $314,30 \pm 23,30$ & $315,05 \pm 26,10$ & C. gracilis & Laboratorio \\
\hline & & $370,00 \pm 20,00$ & $356,00 \pm 15,10$ & Mezcla & Laboratorio \\
\hline & & $320,00 \pm 23,30$ & $378,00 \pm 22,05$ & C. gracilis & Exterior \\
\hline & & $326,10 \pm 24,08$ & $362,00 \pm 12,10$ & Mezcla & Exterior \\
\hline \multirow[t]{2}{*}{ (7 días) } & $34 \pm 0,14$ & & & C. gracilis & Laboratorio \\
\hline & $27 \pm 0,60$ & & & Mezcla & Exterior \\
\hline \multirow{6}{*}{$\begin{array}{l}\text { Pluteus } 6 \text { brazos } \\
\quad \text { (12 días) }\end{array}$} & & $860,00 \pm 0,00$ & $740,00 \pm 0,00$ & C. gracilis & Laboratorio \\
\hline & & $640,00 \pm 0,00$ & $600,00 \pm 0,00$ & Mezcla & Laboratorio \\
\hline & & $780,00 \pm 0,00$ & $600,00 \pm 0,00$ & C. gracilis & Exterior \\
\hline & & $840,00 \pm 0,00$ & $960,00 \pm 0,00$ & Mezcla & Exterior \\
\hline & $8,00 \pm 0,80$ & & & Ambas & Exterior \\
\hline & $10,00 \pm 0,12$ & & & Ambas & Laboratorio \\
\hline \multirow{6}{*}{$\begin{array}{l}\text { Larva competente } \\
\text { (14 dias) }\end{array}$} & & $880,00 \pm 19,20$ & $780,00 \pm 14,30$ & C. gracilis & Laboratorio \\
\hline & & $820,00 \pm 16,12$ & $660,00 \pm 10,20$ & Mezcla & Laboratorio \\
\hline & & $820,00 \pm 16,10$ & $680,00 \pm 11,30$ & C. gracilis & Exterior \\
\hline & & $880,00 \pm 12,10$ & $980,00 \pm 6,09$ & Mezcla & Exterior \\
\hline & $5,00 \pm 0,00$ & & & Mezcla & Exterior \\
\hline & $10,00 \pm 0,00$ & & & Mezcla & Laboratorio \\
\hline (16 días) & & $900,00 \pm 1,90$ & & C. gracilis & Laboratorio \\
\hline \multirow[t]{2}{*}{ (18 días) } & & $760,00 \pm 0,99$ & & C. gracilis & Laboratorio \\
\hline & & $740,00 \pm 0,77$ & & Ambas & Exterior \\
\hline \multirow{2}{*}{$(20$ días) $*$} & $5,00 \pm 0,00$ & & & Mezcla & Exterior \\
\hline & $10,00 \pm 0,00$ & & & Mezcla & Laboratorio \\
\hline $\begin{array}{l}\text { Larva metamórfica } \\
(21,22 \text { y } 23 \text { días })\end{array}$ & & $960,00 \pm 9,96$ & & Mezcla & Exterior \\
\hline Juveniles & & $1100,00 \pm 22,74$ & & C. gracilis & Laboratorio \\
\hline (24 días) & & $1040,00 \pm 9,96$ & & C. gracilis & Exterior \\
\hline
\end{tabular}

* datos significativos

cultivadas a temperatura de $29^{\circ} \mathrm{C}$ y alimentadas con $C$. gracilis, la longitud del cuerpo fue de 320,30 $\pm 23,30$ $\mu \mathrm{m}$ y la longitud de los brazos fue de $378 \pm 22,05 \mu \mathrm{m}, \mathrm{y}$ para la mezcla fue de $326,10 \pm 24,08 \mu \mathrm{m}$ y $362 \pm 12,10$ $\mu \mathrm{m}$ respectivamente (Tabla 1 ).

El mayor porcentaje promedio de supervivencia a los siete días con la mezcla de C. gracilis e I. galbana fue de $22 \pm 0,60 \%$ a temperatura exterior y para las larvas alimentadas con $C$. gracilis fue de $34 \pm 0,14 \%$ a temperatura del laboratorio. Entre los días 9, 10 y 11 no se detectaron diferencias significativas $(P>0,05)$ en la morfología de las larvas de $T$. ventricosus permaneciendo en el estadio de cuatro brazos.
El día 12 se observaron larvas con seis brazos en los tratamientos de ambas temperaturas, alimentadas con $C$. gracilis y la mezcla de microalgas. La longitud del cuerpo de las larvas mantenidas a temperatura del laboratorio fue de $860 \pm 0,00 \mu \mathrm{m}$ con $C$. gracilis y con la mezcla fue de $640 \pm 0,00 \mu \mathrm{m}$. A temperatura exterior las larvas alimentadas con $C$. gracilis tuvieron una longitud del cuerpo de $780 \pm 0,00 \mu \mathrm{m}$ mientras que en las alimentadas con la mezcla fue de $840 \pm 0,00 \mu \mathrm{m}$ (Tabla 1). El mayor porcentaje promedio de supervivencia para este estadio con la mezcla de microalgas fue de 8,00 $\pm 0,80$ a temperatura exterior y de $10,00 \pm 0,12 \mu \mathrm{m}$ a temperatura del laboratorio (Tabla 1). 


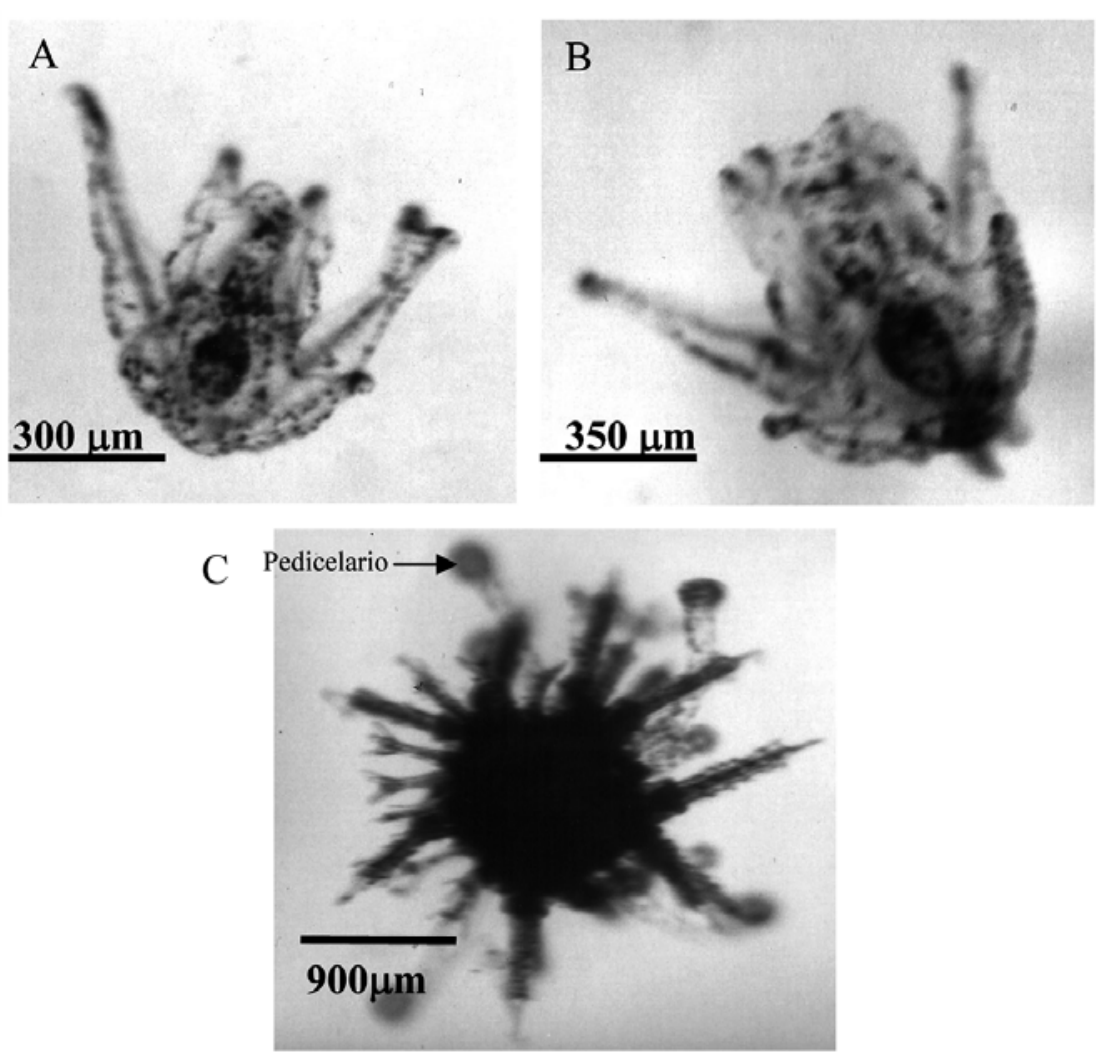

Figura 2

Larvas de Tripneutes ventricosus. A) cuatro brazos, B) ocho brazos (inicio del estadio competente), C) Juvenil

Tripneutes ventricosus larvae. A) Four arms larva, B) Eight arms larva (initial competent stage), C) Juvenile

\section{Periodo competente}

El día 14 se observaron larvas de 8 brazos (Fig. 2B) en el tratamiento con temperatura del laboratorio y alimentadas con C. gracilis, con una longitud del cuerpo de $880 \pm$ $19,20 \mu \mathrm{m}$ y $780 \pm 14,30 \mu \mathrm{m}$ de abertura entre los brazos respectivamente; cuando las larvas se alimentaron con la mezcla de microalgas, la longitud del cuerpo fue de $820 \pm$ $16,12 \mu \mathrm{m}$ y $660 \pm 10,20 \mu \mathrm{m}$ de abertura de los brazos (Tabla 1).

Las larvas cultivadas a temperatura del exterior y alimentadas con $C$. gracilis presentaron una longitud del cuerpo de $820 \pm 16,10 \mu \mathrm{m}$ y las alimentadas con la mezcla, de $880 \pm 12,10 \mu \mathrm{m}$; la mayor abertura de los brazos fue de $680 \pm 1130 \mu \mathrm{m}$ en las larvas alimentadas con $C$. gracilis y de $980 \pm 12,20 \mu \mathrm{m}$ en las alimentadas con la mezcla. La disminución de los primeros brazos indicó el inicio del periodo competente.
El mayor porcentaje promedio de supervivencia de las larvas a los 14 días, fue de $20 \pm 0,70 \%$, a temperatura del laboratorio y alimentadas con la mezcla de C. gracilis e I. galbana.

Durante los días 15 y 16, las larvas se mantuvieron en el estadio de ocho brazos. La longitud de cuerpo de las larvas alimentadas con C. gracilis fue de $900 \pm 1,90 \mu \mathrm{m}$, a temperatura del laboratorio, el día 16, siendo este valor ligeramente mayor al obtenido en las larvas cultivadas a temperatura del exterior y alimentadas con la mezcla de microalgas $(880 \pm 0,00)$. La supervivencia no mostró diferencias significativas (ANOVA, $\mathrm{F}=1,87 ; P>0,05$ ).

Durante los días 17 y 18, se observó la absorción de todos los brazos y la disminución del volumen del cuerpo. A los 18 días, la longitud de las larvas alimentadas con C. gracilis a temperatura del laboratorio fue de $760 \pm 0,99$ 
$\mu \mathrm{m}$ (Tabla 1) y la de las larvas alimentadas con la mezcla a temperatura del exterior fue de $740 \pm 0,77 \mu \mathrm{m}$.

Durante los días 19 y 20, las larvas se mantuvieron en estado competente en todas las condiciones experimentales. En relación a la supervivencia hubo diferencias significativas (ANOVA, $\mathrm{F}=3,19 ; P<0,05$ ) en la interacción día - dieta, detectándose mediante la prueba a posteriori (LSD), $P<0,05$ ), que esta diferencia ocurrió el día 20 en las larvas alimentadas con ambas dietas, con los mayores valores promedios de 5,00 $\pm 0,00$ y $10,00 \pm 0,00 \%$ a temperatura exterior y de laboratorio, respectivamente.

\section{Periodo metamórfico}

Durante los días 21, 22 y 23 se observó el mayor número de postlarvas fijadas al fondo de los recipientes de cultivo en ambas temperaturas, con el mayor valor de longitud del cuerpo de 960,00 $\pm 0,00$ en las larvas alimentadas con la mezcla de microalgas a temperatura exterior. El día 24 concluyó la metamorfosis, llegando hasta la exotropía de las postlarvas del erizo T. ventricosus (Fig. 2C). El mayor valor de longitud del cuerpo fue $1100 \pm$ 22,74 y $1040 \pm 0,00 \mu \mathrm{m}$ en juveniles alimentados con $C$. gracilis a temperatura del laboratorio y exterior respectivamente (Tabla 1 ).

\section{Discusión}

\section{Desarrollo larvario}

La variación del ancho de la testa (109 a $136 \mathrm{~mm})$ de los ejemplares sexualmente maduros de Tripneutes ventricosus utilizados en esta investigación estuvo muy cercana a los valores señalados por Caso (1974) y Liao \& Clark (1995) quienes señalaron tallas de $108 \mathrm{~mm}$ y $160 \mathrm{~mm}$ respectivamente en individuos maduros sexualmente.

El tiempo de desarrollo embrionario de T. ventricosus fue de 24 horas, superior al tiempo registrado para Echinometra lucunter por Astudillo et al. (2005). Posteriormente se inició la fase de prisma, característica de la clase Echinoidea (Ruppert \& Barnes 1996). En el inicio de este estadio, el lado ventral de la larva se transformó convirtiéndose en una superficie oral aplanada y en la zona dorsal se desarrolló una zona abultada y curva como lo refiere Okasaki (1975).

Las especies y cantidad de microalgas suministradas como alimento a $T$. ventricosus, corresponden a las empleadas en el cultivo de Arbacia punctulata (Chaetoceros gracilis e Isochrysis galbana entre 20000 a 80000 cél mL $\mathrm{mL}^{-1}$ ) según García et al. (2005). Asimismo, Buitrago et al. (2003) en investigaciones con Lytechinus variegatus emplearon la microalga $C$. muelleri en concentraciones de 20000 cél $\mathrm{mL}^{-1}$; al respecto, Abalde et al. (1998) mencionan que el uso de diferentes especies de microalgas en el cultivo de organismos marinos, es una estrategia para cubrir sus exigencias nutricionales. Aunque Hadfield et al. (2001) indican que en el laboratorio, el régimen alimenticio con una o pocas especies de microalgas restringe la variedad de nutrientes que está presente en el alimento y no permite los cambios en la dieta que ocurren en la naturaleza a medida que la larva crece.

El tiempo de aparición de las larvas de dos brazos (tercer día) en este estudio, fue superior al registrado para E. lucunter cultivado a $28,1 \pm 1,4^{\circ} \mathrm{C}$ (Astudillo et al. 2005), para A. punctulata cultivado a temperatura exterior de $30 \pm 1^{\circ} \mathrm{C}$ (García et al. 2005) y para L. variegatus cultivado a $28 \pm 2{ }^{\circ} \mathrm{C}$ (Gómez \& Gómez 2005). Posiblemente esta diferencia se debió a tres factores: la localidad y época del año (agosto) en que se recolectaron los erizos reproductores (Amy 1983), la calidad de las microalgas utilizadas (Beddingfield \& McClintock 1998), o a la especie de erizo cultivada (Eckert 1998).

Las larvas de T. ventricosus presentaron cuatro brazos al sexto día; tiempo que fue superior para la producción de estas larvas en otras especies de erizos tropicales (Bustos et al. 2001). La diferencia cronológica pudo estar relacionada con la concentración del alimento, la cual es determinante para la exitosa progresión de ciertos estadios larvales en equinodermos (Metaxas \& Young 1998), o a características propias de las diferentes especies comparadas, ya que las temperaturas empleadas estuvieron muy cercanas a las utilizadas en este estudio y en general variaron entre los 25 a $31^{\circ} \mathrm{C}$. La característica más resaltante en el estadio de cuatro brazos fue la aparición de protuberancias en ambos lados de la parte inferior de la larva, las cuales probablemente sean los esbozos de los brazos posterolaterales. Estructuras equivalentes fueron observadas en el erizo A. punctulata pero a nivel de los brazos (García et al. 2005).

Las larvas de seis brazos observadas a los 12 días, mantuvieron en el estómago la pigmentación del alimento ingerido coincidiendo con lo referido por Gómez \& Gómez (2005). Por otra parte, en el cultivo realizado con el erizo Arbacia punctulata, las larvas presentaron una pigmentación rojo intenso en los extremos de los brazos (García et al. 2005), no observada en este estudio, siendo referido por Jong-Westman et al. (1995) que la carencia o ausencia de coloración en las larvas puede estar relacionada con su estado de salud.

El tiempo de desarrollo de los ocho brazos en todos los tratamientos (14 días) fue superior al establecido por García et al. (2005) para A. punctulata (7-10 días) y por 
Astudillo et al. (2005) para E. lucunter (8 días). Esta diferencia cronológica puede estar relacionada con el bajo contenido nutricional que aportaron las dietas suministradas. Posiblemente C. gracilis y la mezcla $C$. gracilis más I. galbana no poseían suficientes ácidos grasos para cubrir la demanda requerida por las larvas en estas etapas del ciclo de vida. Al respecto Cabrera (1993) menciona que los ácidos grasos son esenciales para la dieta de muchos animales acuáticos. Por otro lado, Hart \& Strathmann (1994) indican que el tamaño de las células de las dietas empleadas es otro elemento determinante para el buen desarrollo y estado de salud de las larvas. El día 14 en la larva de ocho brazos se inició el período competente, observándose la presencia del rudimento juvenil, coincidiendo con Salas-Garzas et al. (2005) para el erizo rojo Strogylocentrotus franciscanus; igualmente entre los días 17 y 18 días, se reabsorbieron todos los brazos marcando el período metamórfico, proceso que es normal en la mayoría de los equinoideos (Fuentes \& Barros 2000, Young \& George 2000), y definido por Hardfield et al. (2001) como la pérdida de las estructuras larvales para producirse el rudimento juvenil bien desarrollado.

Entre los días 21 y 23 se observaron las primeras larvas fijadas en el fondo y en las paredes de los envases, caracterizándose por su fase endotrófica que puede durar entre 4 y 8 días, dependiendo de la especie (Fuentes \& Barros 2000). El día 24 se observaron las primeras postlarvas de $T$. ventricosus con características distintivas del erizo juvenil y adulto. Para que las larvas puedan cumplir con todos los requerimientos del proceso de metamorfosis, necesitan buena calidad de agua (Bustos \& Olave 2001), concentración de alimento adecuada (Metaxas \& Young 1998), requerimiento nutritivo (Sheperd \& Bromage 1998) y el adecuado inductor (microalga bentónica, como son los casos de Amphora sp. y Navicula sp.) con lo cual se garantiza el éxito de la fijación de larvas competentes alcanzando el periodo metamórfico (Gómez \& Gómez 2005). Sin embargo, en este estudio no se utilizó inductor, por lo que la metamorfosis no ocurrió al mismo tiempo en todas las larvas, observándose el día 24 algunas larvas con presencia de brazos; al respecto Michel (1985) refiere que hasta dos semanas después de la metamorfosis del primer erizo se pueden observar larvas en proceso de cambio.

\section{Supervivencia larval}

Rupert \& Barnes (1996) señalan que durante la fase prisma se obtienen altos porcentajes de supervivencia debido a que aún las larvas dependen de su reserva, lo cual fue corroborado en este estudio con un $100 \%$ de supervivencia, sin embargo, tres días después disminuyó un $39 \pm 0,11 \%$ en larvas alimentadas con las dos dietas en ambas temperaturas, lo cual podría ser atribuido a que el contenido nutricional de las microalgas varía dependiendo del medio de cultivo en el que se desarrollen y que cada especie de erizo posee su propio requerimiento nutricional (Brown 1991, Silva 1999). Los porcentajes de supervivencia no mostraron diferencias antes de iniciarse el periodo competente en larvas alimentadas con ambas dietas a temperatura del laboratorio, indicando que C. gracilis suministrada como dieta monoalgal proporcionó un buen aporte nutricional a las larvas. Al respecto, Kitamura et al. (1993) utilizaron C. gracilis en la alimentación de Pseudocentrotus depressus y Anthocidaris crassispina. Igualmente, Bustos et al. 1991) y Zamora \& Stoltz (1994) establecieron que C. gracilis fue la dieta con la cual se han logrado los mejores resultados en la alimentación larval de Loxechinus albus, debido a su tamaño y calidad nutricional. En el erizo Echinometra lucunter se logró una mayor supervivencia utilizando C. gracilis y alcanzó el estadio postlarval en 12 días (Astudillo et al. 2005).

Por otra parte, Roller \& Stickle (1993) indican que la temperatura es un factor que controla el desarrollo de los primeros estadios larvales no sólo de Lytechinus variegatus, sino de la mayoría de las especies de erizos; igualmente Beddingfield \& McClintock (1998) afirman que las bajas tasas de alimentación pueden contribuir a un déficit en los nutrientes requeridos por las larvas, ocasionando una baja en la supervivencia, en este estadio pareciera evidenciarse que las supervivencia larval fue favorecida por la mezcla de microalgas y la temperatura exterior.

Hasta el día 14 el mayor porcentaje de supervivencia de Tripneutes ventricosus $(8,00 \pm 0,80$ y $10,00 \pm 0,12)$ con la mezcla de microalgas a temperatura del exterior y laboratorio respectivamente, fue menor a los valores obtenidos por García et al. (2005) en las larvas de $A$. punctulata con C. gracilis $(21,66 \%)$ e I. galbana $(23,33 \%)$, pero similares a los obtenidos por Astudillo et al. (2005) en larvas de Echinometra lucunter, con valores entre $14,7 \pm 3,80$ con $C$. gracilis y $14,00 \pm 2,60$ con la mezcla de C. gracilis e I. galbana. Metaxas \& Young (1998) indicaron los efectos positivos en el desarrollo larval de E. lucunter cuando alimentaron con una dieta mixta constituida por I. galbana, Dunaliella tertiolecta y Thalassiosira sp.

La disminución del porcentaje de supervivencia a los 12 días con ambas dietas en las dos temperaturas puede ser atribuida a que las larvas iniciaron el estadio competente para abandonar sus hábitos planctónicos 
para establecerse en el fondo y paredes de los recipientes de cultivo. Al respecto, McEdward \& Herrera (1999) afirman que pocos recambios de agua y altas densidades de larvas pueden contribuir a una pobre supervivencia y un lento desarrollo del erizo. Estadísticamente, con el transcurso de los días, se mantuvo el mismo porcentaje de supervivencia tanto para los erizos alimentados con C. gracilis como con la mezcla de C. gracilis e I. galbana en ambas temperaturas.

Finalmente en el día 20 se detectaron las diferencias en los porcentajes de supervivencia en las larvas alimentadas con la mezcla de microalgas a temperatura del exterior respecto a la dieta monoalgal. Esta diferencia ha sido referida por algunos autores destacando las ventajas del uso de dietas mixtas para el cultivo de larvas de equinodermos, ya que satisfacen mejor sus requerimientos nutricionales (Metaxas \& Young 1998). $\mathrm{Si}$ se considera que las larvas asimilan completamente la variedad de especies de microalgas ingeridas, se puede explicar por qué las dietas monoalgales no satisfacen de igual forma los requerimientos para el crecimiento y desarrollo larvario (Fonseca 2001), aunque Hardfield et al. (2001) refieren que en condiciones experimentales no se logra suplir completamente los nutrientes requeridos por las larvas a medida que crecen en comparación con lo que ocurre en la naturaleza.

Durante los días 20 y 21 en larvas alimentadas con la mezcla de C. gracilis e I. galbana en ambas temperaturas, fue difícil determinar la supervivencia debido a que las larvas se encontraban en proceso metamórfico o fijadas a las paredes y fondo de los recipientes de cultivo.

\section{Longitud del cuerpo}

En cuanto a la longitud del cuerpo de las larvas no hubo diferencias en ambas temperaturas y dietas estudiadas. En el estadio de dos brazos el mayor valor promedio a temperatura variable y alimentadas con C. gracilis, fue superior al indicado por Gómez \& Gómez (2005) en larvas de dos brazos de Lytechinus variegatus alimentadas con Tetraselmis chuii e I. galbana, mientras que estuvo cercano a los valores obtenidos por Astudillo et al. (2005) en larvas de $E$. lucunter $(182,4 \pm 14,06 \mu \mathrm{m})$ alimentadas con I. galbana y en larvas de A. punctulata (152 a 266 $\mu \mathrm{m})$ cuando se alimentaron con C. gracilis, C. calcitrans, I. galbana, Nannochloropsis oculata y T. chuii (García et al. 2005). En este estudio la longitud del cuerpo disminuyó en todos los tratamientos a partir del día 18, debido a la reabsorción de los brazos o inicio del período metamórfico (Gómez \& Gómez 2005). Los juveniles del erizo T. ventricosus alimentados con C. gracilis a temperatura del laboratorio mostraron una mayor longitud del cuerpo $(1100 \pm 22,74 \mu \mathrm{m})$ que a temperatura del exterior. Sheperd \& Bromage (1998) afirman que esto es influenciado por la calidad de la dieta suministrada, en términos del balance nutritivo y contenido energético requerido por las larvas en esta fase de desarrollo. Otra causa de esta diferencia en la longitud del cuerpo pudo estar relacionado con que $C$. gracilis es la microalga con mejor tamaño y adaptabilidad a las condiciones de cultivo de organismos marinos de importancia económica (Rodríguez 2000).

En resumen, el tiempo de desarrollo embrionario del erizo Tripneutes ventricosus fue de 24 horas, completándose el desarrollo larvario hasta la metamorfosis en 24 días a partir de la fecundación para ambas temperaturas y dietas. No se detectaron diferencias morfológicas en la longitud del cuerpo entre los tratamientos, obteniéndose los máximos valores $(1100 \pm$ $22,74 \mu \mathrm{m}$ ) en las larvas alimentadas con la mezcla de microalgas a temperatura del laboratorio; sin embargo, para las mismas condiciones de cultivo la supervivencia fue significativa con valores de $10 \%$ hacia finales del cultivo.

\section{Agradecimientos}

Agradecemos a los distinguidos evaluadores por la lectura crítica del manuscrito, lo que permitió mejorarlo.

\section{Literatura citada}

Abalde J, A Cid, J Fidalgo, E Torres \& C Herrero. 1998. Microalgas: Cultivo y aplicaciones, pp. 159-161. Universidad da Coruña, Coruña.

Amy R. 1983. Gametes sizes and development time tables of five tropical sea urchins. Bulletin of Marine Science 33: 173-176.

Astudillo D, J Rosas, A Velásquez, T Cabrera \& C Maneiro. 2005. Crecimiento y supervivencia de larvas de Echinometra lucunter (Echinoidea: Echinometridae) alimentadas con las microalgas Chaetoceros gracilis e Isochrysis galbana. Revista de Biología Tropical 53 (3/4): 337-344.

Beddingfield S \& J McClintock. 1998. Differential survivorship, reproduction, growth and nutrient allocation in the regular echinoid Lytechinus variegates (Lamarck) fed natural diets. Journal of Experimental Marine Biology and Ecology 226: 195-215.

Brown MR. 1991. The amino-acid and sugar composition of 16 species of microalgae used in mariculture. Journal of Experimental Marine Biology and Ecology 145: 79-99.

Buitrago E, C Lodeiros, C Lunar, F Indorf, K Frontado, M Pulido \& Z Vasquez. 2003. Efecto de la densidad larvaria sobre el desarrollo, crecimiento y supervivencia del erizo Litechynus variegatus (Echinodermata: Echinoidea). $31^{\text {st }}$ Scientific Meeting of the Association of Marine Laboratory of the de Caribbean, Trinidad and Tobago, pp. 155-160. 
Bustos E \& S Olave. 2001. El cultivo del erizo Loxechinus albus, 22 pp. Informe Técnico presentado al FONDEF. Instituto de Fomento Pesquero de Chile, Valparaíso.

Bustos E, C Godoy, S Olave \& R Troncoso. 1991. Desarrollo de técnicas de producción de semillas y repoblación de recursos bentónicos. I. Investigaciones en el erizo cghileno (Loxechinus albus (Molina, 1782), pp. 61-138. Instituto de Fomento Pesquero de Chile, Santiago.

Cabrera T. 1993. The nutritional value of live feeds and egg quality on the larval growth and survival of flounder (Paralichthys olivaceus Temminck et Schlegel). PhD dissertation, Pusan University, Pusan, Korea, 164 pp.

Caso ME 1974. Contribución al estudio de los equinoideos de México el género, Tripneutes agassiz, morfología y ecología de Tripneutes ventricosus (Lamarck). Anales del Centro de Ciencias del Mar y Limnología 1(1): 1-21.

Domínguez A, J Rosas, A Velásquez, T Cabrera \& E Mata. 2007. Desarrollo, supervivencia y crecimiento del erizo Lytechinus variegatus (Lamarck, 1816) (Echinodermata: Echinoidea) alimentado con microalgas a dos salinidades y temperaturas diferentes. Revista de Biología Marina y Oceanografía 42(1): 49-57.

Eckert G. 1998. Larval development, growth and morphology of the sea urchin Diadema antillarum. Bulletin of Marine Science 63(2): 443-451.

Fonseca MJ. 2001. Efecto de seis dietas microalgales en el desarrollo larvario, metamorfosis y obtención de juveniles del erizo morado Strongylocentrotus purpuratus (Stimpson), $83 \mathrm{pp}$. Instituto de Investigaciones Oceanológicas, Facultad de Ciencias Marinas, Universidad Autónoma de Baja California, Baja California, México, 83 pp.

Fuentes I \& C Barros. 2000. Larval development and metamorphosis of cultured Tetrapygus niger (Echinodermata: Echinoidea). An uncommon form of echinoplutei. Invertebrate Reproduction and Development 37(3): 201-209.

García M, J Rosas, I Hernández, A Velásquez \& T Cabrera. 2005. Supervivencia y crecimiento larval de Arbacia punctulata (Echinodermata: Echinoidea) alimentada con cinco microalgas a dos salinidades. Revista de Biología Tropical 53(3/4): 329-336.

Gómez A. 2000. Abundancia de Litechynus variegatus (Echinoidea: Toxopneustidae) en la Isla de Cubagua, Venezuela. Revista de Biología Tropical 48(1): 125-131.

Gómez O \& A Gómez. 2005. Desarrollo embrionario y larval de Lytechinus variegatus (Lamarck) (Echinodermata: Echinoidea: Toxopneustidae) en condiciones de laboratorio en la Isla de Margarita-Venezuela. Revista de Biología Tropical 53 (Suppl. 3): 313-318.

Gordon H, J Miller, D Pawson \& P Kier. 1995. Echinoderms of Florida and the Caribbean Sea Stars, Sea Urchins and Allies, 390 pp. Smithsonian Institution Press, Washington.

Grosjean P, C Spirlet, P Gosselin, D Vaïtilingon \& M Jangoux. 1998. Land-based, closed-cycle echinoculture of Paracentrotus lividus (Lamarck) (Echinoidea:
Echinodermata): A long-term experiment at a pilot scale. Journal of Shellfish Research 17: 1523-1531.

Hardfield M, E Carpizo-Ituarte, K del Carmen \& B Nedved. 2001. Metamorphic competence, a major adaptive convergence in marine invertebrate larvae. American Zoology 41: 1123-1131.

Hart MW \& RR Strathmann. 1994. Functional consequences of phenotypic plasticity in echinoids larvae. Marine Biology 117: 615-622.

Jong-Westman M, P Qian, B March \& T Carefoot. 1995. Artificial diets in sea urchin culture: Effects of dietary protein level and other additives on egg quality, larval morphometrics, and larval survival in the green sea urchin, Strongylocentrotus droebachiensis. Canadian Journal of Zoology 73: 2080-2090.

Kitamura H, S Kitahara \& HB Koh. 1993. The induction of larval settlement and metamorphosis of two sea urchins, Pseudocentrotus depressus and Anthocidaris crassispina, by free fatty acids extracted the coralline red alga Coralline pilulifera. Marine Biology 115: 387-392.

Klumpp D, JT Salita-Espinosa \& MD Fortes. 1993. Feeding ecology and trophic role of sea urchins in a tropical seagrass community. Aquatic Botany 45: 205-229.

Lawrence AL \& JM Lawrence. 2003. Importance status and future research needs for formulated feeds for sea urchin Aquaculture Fisheries and Ecology. Proceedings of the International Conference on Sea-Urchin Fisheries and Aquaculture, pp. 275-283. Universidad Católica del Norte, Puerto Varas.

Lawrence JM, D Lawrence, S McBride, S George, S Watts \& L Plank. 2001. Development in the use of prepared feeds in sea urchin Aquaculture. World Aquaculture 32(3): 3439.

Liao Y \& AM Clark. 1995. The echinoderms of southern China, 484 pp. Science Press, Beijing.

MacEdward L \& C Herrera. 1999. Larval spicules, cilia, and symmetry as remnants of indirect development in the direct developing sea urchin Heliocidaris erythrogramma. Developmental Biology 167: 405-415.

Metaxas A \& CM Young. 1998. Behavior of echinoids larvae arond sharp haloclines: effects of the salinity gradient and dietary conditioning. Marine Biology 131: 291-305.

Michel H. 1985. Culture of Lytechinus variegatus (Lamarck) (Echinodermata: Echinoidea) from egg to young adults. Bulletin of Marine Science 34: 312-314.

Okasaki K. 1975. Normal development to metamorphosis. World Aquaculture 7: 177-231.

Pacheco E. 1998. Cultivos acuícolas no tradicionales: Erizo. Panorama Acuícola 3(5): 36-37.

Rodríguez J. 2000. Valores nutricionales de las microalgas. Boletín del Instituto Oceanográfico 28(3/4): 234-250.

Roller R \& W Stickle. 1993. Effects of temperature and salinity acclimation of adults on larval survival, physiology, and early development of Lytechinus variegatus (Echinodermata: Echinoidea). Marine Biology 116: 583-591. 
Ruppert A \& D Barnes. 1996. Zoología de los invertebrados, 1114 pp. McGraw-Hill Interamericana, Barcelona.

Salas-Garza A, E Carpizo-Ituarte, G Parés-Sierra, R Martínez-López \& R Quintana-Rodríguez. 2005. Producción de juveniles de erizo rojo Strongylocentrotus franciscanus (Echinodermata: Echinoidea) en Baja California, México. Revista de Biología Tropical 53 (Suppl. 3): 345-355.

Sheperd J \& N Bromage. 1998. Nutrition and growth in intensive fish farming, 159 pp. BSP Professional Books, Oxford.

Silva A. 1999. Efecto de la microalga Isochrysis galbana en el cultivo temprano de larvas de Paralichthys adspersus. Ciencias Marinas 25(2): 267-276.
Sokal R \& F Rohlf. 1981. Biometría, principios y métodos estadísticos en la investigación biológica, 832 pp. H. Blume, Madrid.

Young C \& S George. 2000. Larval development of the tropical deep-sea echinoid Aspidodiadema jacobyi. Phylogenetic implications. The Biological Bulletin 198: 387-395.

Zamora S \& W Stotz. 1994. Cultivo masivo en laboratorio de juveniles de erizo Loxechinus albus (Molina, 1782), (Echinodermata:Echinoidea). Investigación Pesquera, (Chile) 38: 37-54 .

Recibido el 28 de mayo de 2008 y aceptado el 30 de abril de 2009 